\title{
A Low Energy Uneven Cluster Protocol Design for Wireless Sensor Network
}

\author{
Yi Liu, Shan Zhong, Licai You, Bu Lv, Lin Du \\ Computer Science and Engineering College, Changshu Institute of Technology, Changshu, China \\ Email: sunshine-620@163.com
}

Received October 28, 2011; revised December 24, 2011; accepted January 12, 2012

\begin{abstract}
A low energy uneven cluster protocol design method is proposed. Aiming at the random choosing for cluster head of traditional Leach protocol, and the defect of the single hop from all the cluster heads to the sink node, an improved method for Leave protocol is advanced. Firstly, the election model of cluster head is improved, and the node residual energy is considered in the process of threshold and the cluster head election to improve the whole network life circle. In the multi-hop route, choosing the maximum energy and the nearest node as the next hop and a route transferring data among many clusters is formed. The experiment shows the method having great improvement compared with Leach protocol and prolonging the network life cycle.
\end{abstract}

Keywords: Cluster; Threshold; Leach Protocol; Low Energy

\section{Introduction}

Wireless sensor network is deployed in the monitoring area by a large number of micro sensor nodes, and the nodes using the wireless communication form a wireless ad hoc network. Every sensor node is composed of sensing unit, calculating unit, communication unit and storage unit. As sensor network has the characters of low cost and low power, it can monitor and sense the environmental objects, through the collection and processing, transferring the information of control to the users. Currently, wireless sensor network has widely application prospect in military application, medical health, and the commercial application.

WSN routing protocol is the main research, because the sensor nodes are battery powered, so the capacity is very limited and very difficult to replace the battery. Therefore, the energy saving of route protocol for wireless sensor network is the current research spot. Cluster route protocol is a low energy route protocol, and it separate the whole WSN network into several areas, these areas are regards as cluster. All the clusters are composed by cluster head and the ordinary node. Cluster node is responsible for collecting data and data fusion, while the ordinary node of the cluster is responsible for sending the data to the cluster head.

Currently, there are a lot of research about the WSN low-power protocols, the first distributed cluster protocol is Leach protocol $[1,2]$, and the new cluster protocol is proposed such as Leach-C [3], Teen [4] and Heed [5].
There are also some works for the improved Leach protocols [6-9], The literature [6] proposed a steady group clustering hierarchy algorism, it firstly divide the network into groups, and then directly change the group into the cluster. But it does not consider the multi-hop between the cluster heads. The literature [7] introduced a kind of adaptive dynamic multiple hops non-uniform clustering algorism.

\section{Leach Protocol}

LEACH is the first proposed wireless hierarchical routing protocol, and the "round" concept is defined. Every round can be divided into clusters forming and the steady data transferring phase. In the clusters forming phase, all the nodes of wireless sensor network generated a random value between 0 and 1 , and the threshold $T(n)$ is defined. If the random value of node is larger than threshold, and the node has not been the cluster head in former $1 / p$ round, then the node can be the cluster head.

The threshold of $T(n)$ can be defined as Equation (1):

$$
T(n)= \begin{cases}\frac{p}{1-p(r \bmod 1 / p)} & (n \in G) \\ 0 & (n \notin G)\end{cases}
$$

In Equation (1), $p$ is the expect percentage to become the cluster head, $r$ is the current round, and $G$ is the node set having not been the cluster head in the former $1 / p$ round.

When the ordinary node becomes the cluster head, it 
will broadcast message to the whole network. The node in the network joins the corresponding cluster according the signal strength and broadcasts joining the cluster message to the cluster head. The cluster head will allocate TDMA to every node of the cluster.

Leach protocol has the following problems:

1) Random selection of cluster head will lead to the uneven distribution of the cluster head, making the cluster uneven, load unbalanced and short network stable time.

2) The amounts of the cluster head are volatile, so it is difficult to achieve the theoretical optimal value.

3) The data transferring between the cluster head nodes and the sink node is using the single-hop transfer model, makes the nearby node at the sink node needing to operate the data fusion and the data transfer between all the cluster heads.

\section{Building for Network and Cluster}

In the network building phrase, the sink node broadcast signal to all the network using some transmit power, and after the node of the network receive the signals, it estimates the distance from to the sink node according the signal strength. The whole network can be divided to a series circle area having different radius, the node far away from the sink node have the larger radius, cluster head is still responsible for receiving and fusing the data of the cluster, and the residential energy [5] of the node is considered in the cluster head to improve the threshold equation showed as Equation (2):

$$
T(n)= \begin{cases}\frac{p}{1-p(r \bmod 1 / p)} \frac{E_{\text {current }}}{E_{\text {initial }}} & (n \in G) \\ 0 & (n \notin G)\end{cases}
$$

The approach makes good use of the node residual energy, improving the probability of high residual energy node to be the cluster head.

The process of competition of cluster head is showed as follows:

1) Each node generate a random value between 0 and 1.

2) If the random value of node is less than threshold, then the node becomes the cluster head and broadcasts the competitive cluster head message in the whole network.

3) When the competitive node received the competitive cluster head message, it will check if the neighbor competitive node array containing the node. If the neighbor competitive node array has not contained the node, the cluster head node is added to the cluster head node array.

4) If all the nodes have not received the competitive cluster head message in a specific time $t$, every competi- tive cluster head node compare the neighbor competitive node posited in the same circle. If they are in the same circle, then the largest energy node in the same circle becomes the cluster head node.

5) When the node becomes the cluster head node, it broadcasts the final cluster head message in the whole network. The node received the final cluster head message judges whether the cluster head node is in its neighbor competitive node array. If it is existed in the neighbor competitive node array, then judges whether they are in the same circle. If they are in the same circle, then the cluster head is removed from the neighbor competitive node array, become the ordinary node and broadcast the ordinary node message. If they are in the same circle, then just removed the node from the neighbor competitive array.

6) The node received the ordinary node message judge whether the ordinary node is in the neighbor competitive node array. If it exists, then remove the node from the neighbor competitive node array.

7) All the ordinary nodes according the final cluster head message send the joining cluster message to cluster head node in the same circle.

\section{Data Transfer Protocol among Clusters}

In order to overcome the shortcomings of the traditional leach protocol, the distance threshold Dist is introduced. If the distance between cluster head and the sink node is less than Dist, then it can communicate with sink node directly. Each node can broadcast the node information message and the message contains the residual energy of node and the distance to the sink node. Cluster head $n_{i}$ receives the message of cluster head $n_{j}$ and the distance between them can be calculated.

The cluster head multi-hop route table is constructed as Equation (3):

$$
\begin{aligned}
n_{i} \cdot \text { Rout }= & \left\{n_{j} \mid \operatorname{dist}\left(n_{j}, \sin k\right)\right. \\
& \left.<\operatorname{dist}\left(n_{i}, \sin k\right) \& \text { neigbor }\left(n_{i}, n_{j}\right)\right\}
\end{aligned}
$$

In Equation (3), the selective route of $n_{i}$ is to choose biggest residual energy node in the selective route node set and the smallest distance node from the sink node. The energy consumption mainly contains the energy consumed in the data fusing and in the data forwarding in the multi-hop. For the former part, if the node in the cluster sends $L$ byte data to the cluster head, then the energy consumed by cluster head is showed as Equation (4):

$$
E_{\text {internal }}=L E_{\text {elec }}+N L E_{\mathrm{DA}}
$$

In the Equation (4), $E_{\text {elec }}$ represents the energy consuming of receiving unit byte data, $E_{\mathrm{DA}}$ represents the 


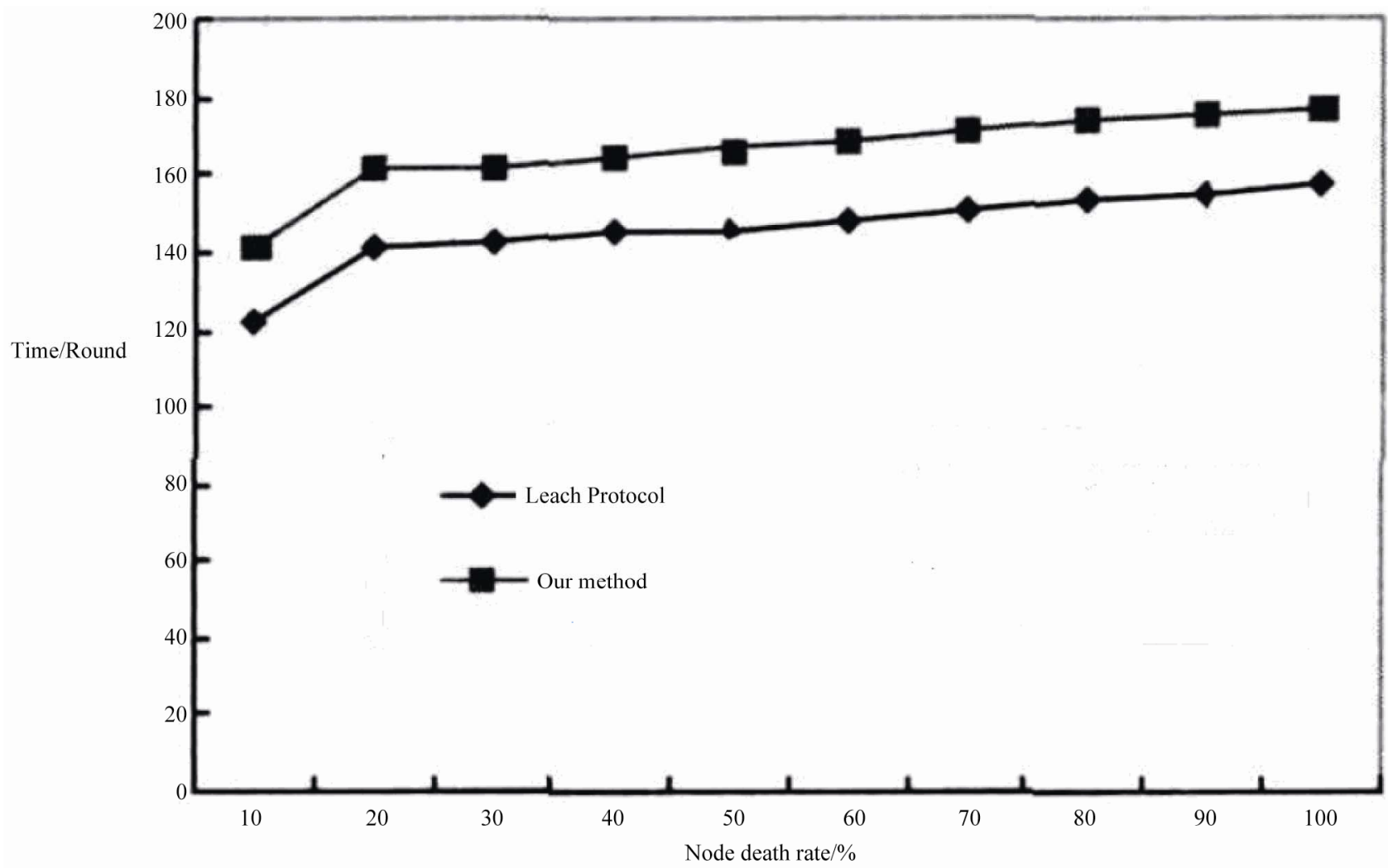

Figure 1. Relation between node death and time.

energy consumption for fusing unit data, where $N$ represents the amount of nodes in the cluster. For the latter part of energy consuming, if cluster head node $n_{i}$ choose the cluster head node $n_{j}$ as the next hop route, $n_{i}$ needs to transfer $L$ byte data to the sink node, and the next hop is the sink node. According to the literature [6], the energy consumed for $n_{i}$ and $n_{j}$ is respectively showed as Equations (5) and (6):

$$
\begin{gathered}
E_{n_{i} \text {-external }}=L E_{\text {elec }}+L \varepsilon_{\text {fs }} \operatorname{dist}\left(n_{i}, n_{j}\right) \\
E_{n_{j} \text {-external }}=2 L E_{\text {elec }}+L \varepsilon_{f s} \operatorname{dist}\left(n_{j}, \sin k\right)
\end{gathered}
$$

In Equation (6), $\varepsilon_{f s}$ is the energy consumed by amplifier, $\operatorname{dist}\left(n_{i}, n_{j}\right)$ represents the distance between the node $n_{i}$ and node $n_{j}$, dist $\left(n_{j}, \sin k\right)$ represents the distance between cluster head node $n_{j}$ and the sink node, $2 L E_{\text {elec }}$ represents the energy consumed for $n_{i}$ receiving $L$ byte data and transferring the data to the sink node.

\section{Simulation Result}

Simulation scenario is as follows: 100 nodes randomly distributed in the different size area, the initial energy of sensor node is $3 \mathrm{~J}$, the size of data packet is $200 \mathrm{bit}$, the size of meta data is 20 bit, the time for transferring the data in every round is $40 \mathrm{~s}$, and the probability for the ordinary becomes the cluster head of the cluster in every round is $8 \%$.

The result of the simulation is showed as Figure 1.

The proposed method compared with the traditional Leach protocol has the time delay priority showed in Figure 1. It is prove our method has the longer life circle than Leach protocol.

\section{Conclusion}

In this paper, based on the research on the Leach protocol, in order to conquer the defect of the Leach protocol, a dynamic, uneven and multi-hop route for wireless sensor network is introduced, and the life circle is improved greatly. The cluster route protocol proposed in this paper considered the energy consumption of cluster head and the energy consumption of multi-hop route. It has the good energy-saving effect. However, the initial parameter of the wireless sensor network such as energy threshold and the distance threshold usually relied on the experience, and it is needed to be trained to get the precise value.

\section{REFERENCES}

[1] W. Heinzelman, A. Chandrakasan and H. Balakrishnan, 
"Energy-Efficient Communication Protocol for Wireless Microsensor Network," Proceedings of the 33rd Annual Hawaii International Conference on System Sciences, Washington DC, January 4-7 2000, pp. 3005-3014.

[2] W. B. Heinzelm, A. P. Chandrakasan and H. Balakrishnan, "An Application Specific Protocol Architecture for Wireless Microsensor Networks," IEEE Transactions on Wireless Communications, Vol. 14, No. 4, 2000, pp. 660670.

[3] A. Manjeshwar and D. P. Agrawal, “TEEN: A Routing Protocol for Enhanced Efficiency in Wireless Sensor Networks," Proceedings of 15th International Parallel and Distributed Processing Symposium, San Francisco, 5 April 2001, pp. 2009-2015.

[4] O. Younis and S. Fahmy, "Heed: A Hybrid, Energy Efficient Distributed Clustering Approach for Ad Hoc Sensor Networks," IEEE Transactions on Mobile Computing, 7-11 March 2004, pp. 366-379.

[5] M. J. Handy, M. Haase and D. Timmermann, "Low Energy Adaptive Clustering Hierarchy with Deterministic
Cluster-Head Selection," 4th International Workshop on Mobile and Wireless Communications Network, Washington, 9-11 September 2002, pp. 368-372.

[6] Y.-M. Hu, Y.-P. Deng and J. Yang, "Load-Balanced Adaptive Group Clustering Algorism for Wireless Sensor Network," Journal of Computer Application, Vol. 8, No. 31, 2011, pp. 2056-2061.

[7] C.-L. Li and C.-H. Hu, “A Dynamic Multi-Hop Non-Uniform Clustering Routing Protocol in Wireless Sensor Networks,” Journal of Central South University (Science and Technology), Vol. 42, No. 7, 2011, pp. 2048-2053.

[8] Y. Hu and J. Wang, "Analysis of LEACH Protocol Based on Ant Algorithm,” Advanced Materials Research, Vol. 24, No. 5, 2011, pp. 2830-2833.

[9] L. Yan, W. Pan, B. Luo, X. Li and J. Liu, "Modified Energy-Efficient Protocol for Wireless Sensor Networks in the Presence of Distributed Optical Fiber Senor Link," IEEE Sensors Journal, Vol. 11, No. 9, 2011, pp. 18151819. 